\title{
Decentralized control techniques for plug-in electric vehicles in MV/LV distribution networks
}

\author{
J. García-Villalobos, I. Zamora, I. Junquera, J.I. San Martín, P. Eguía \\ Department of Electrical Engineering - University of Basque Country - UPV/EHU \\ Alda. Urquijo, s/n, 48013 Bilbao (Spain) \\ e-mail: javier.garciav@ehu.es
}

\begin{abstract}
Decentralized control techniques are acquiring more and more importance to efficiently manage a large number of distributed energy resources (DER). It is expected that, in the future, plug-in electric vehicles (PEVs) will act as DERs providing valued services to power grids. In addition, it has been proven that uncontrolled charging of PEVs will have a significant impact on the operation of $\mathrm{MV} / \mathrm{LV}$ distribution networks. In this context, this paper presents a review on decentralized control approaches to adequately integrate PEVs in MV/LV distribution networks. Among these decentralized control techniques, optimal and droop based controls are the most common. Both have strengths and weaknesses, so, more research about mixing both methods is needed.
\end{abstract}

\section{Keywords}

Plug-in electric vehicles, decentralized control, smart charging, optimized control, droop control.

\section{Introduction}

Currently, PEVs sales are growing in developed countries and it is expected that this trend will continue in the next years/decades [1]. As the number of PEVs increase, MV/LV distribution networks will have to deal with an important increment of energy demand. Uncontrolled charging of PEVs would have undesirable consequences in distribution networks such as voltage deviations, increase of power losses, congestion problems, distribution transformer life expectancy reduction, etc. [2]-[4].

In order to accommodate this new demand, it is necessary to implement an intelligent control to reduce as much as possible the impacts on distribution networks. In addition, smart charging of PEVs will avoid or, at least, delay distribution network upgrades. In this context, two control architectures can be used to implement a smart charging control: the centralized and the decentralized architecture.

In the centralized control architecture, a central controller, aggregator or fleet operator (FO), is in charge of acquiring data from the PEVs and other entities, executing the control algorithm and sending the calculated charging set-point of each PEV under its governance. The central controller has to take into account numerous aspects such as electricity prices, PEVs users' preferences, distribution network status, etc. In this approach, each user will give up the direct control of the charging process of its PEV.

In contrast, the decentralized control, also known as distributed or local control, is based on managing locally the charging process of the PEVs, according to the users' preferences and external data received. This way, users maintain the control of the charging process of their PEVs. As commented before, two main decentralized control techniques have been used to integrate PEVs in distribution networks: the droop control and the optimized control. The first one is widely utilized, focused on voltage and frequency regulation. The second one is based on minimizing or maximizing an objective function.

The aim of this paper is to present the decentralized control approaches used to implement smart charging strategies, as well as identifying the strengths and weaknesses of these approaches. Thus, the characteristics of the decentralized control are presented in Section 2, focusing in the integration of PEVs in distribution networks. Section 3 analyzes the droop control methods used to integrate PEVs that can be found in the literature. Optimized control algorithms are reviewed in Section 4. Section 5 presents some characteristics of the pair PEVmicrogrids. Finally, the main conclusions are presented in Section 6.

\section{Decentralized control characteristics}

In decentralized architectures (Figure 1), the charging control resides in each $\mathrm{PEV}$, i.e. in each owner, rather than in an external entity as the aggregator. This aspect implies that each PEV must have some intelligence implemented. 


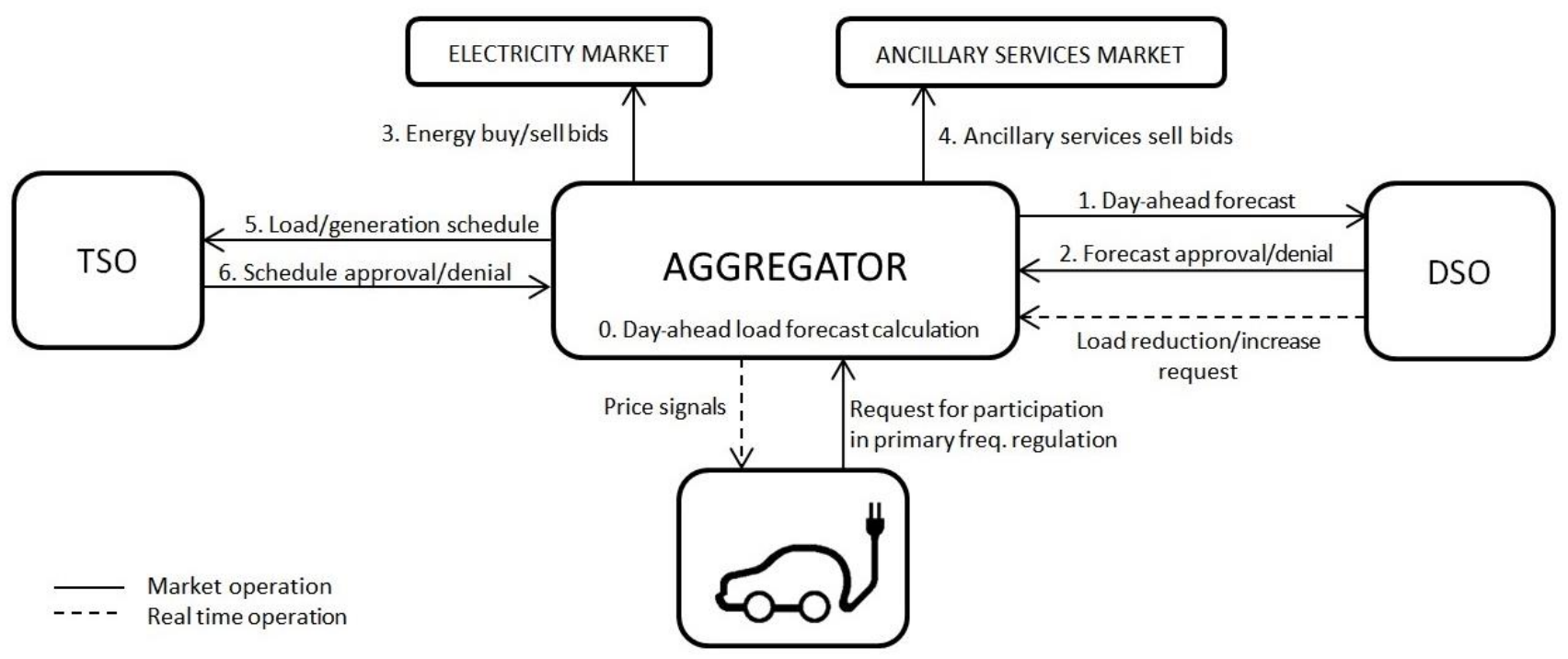

Figure 1. Decentralized control architecture using price signals [5]

Although the decision of "when and how much should be charged" is taken by the PEV/owner itself, there are ways to influence these decisions. One basic way is measuring the local voltage and frequency at the connection node. Depending on these values, charging power can be modulated in order to control the local voltage or provide primary frequency regulation. This technique is known as droop control and it can work without communication infrastructure.

Another way to indirectly control the charging process of PEVs is using price or control signals that can be sent from an aggregator entity or directly from a utility. In this framework, each PEV autonomously seeks to optimize its charging cost, considering the PEV user's preferences. But, the electricity cost will change depending on how much the distribution network is loaded. That is, as energy demand increases, electricity cost will be higher. This way, PEVs energy demand will be shifted from hours where electricity is expensive (highly loaded) to hours where cost is lower (lightly loaded). This optimization process can be carried out without sending private information to external entities, as it happens in the centralized control architectures. Apart from improving privacy, the decentralized architecture present several advantages and drawbacks, as shown in Table 1.

Table 1. Advantages and drawbacks of decentralized control

\begin{tabular}{cl}
\hline \multicolumn{1}{l}{ Advantages } \\
\hline$\checkmark$ & Scalable \\
$\checkmark$ & Improved fault tolerance \\
$\checkmark$ & Less communications infrastructure required \\
$\checkmark$ & Charge control remain in the user \\
$\checkmark$ & Higher consumer acceptance \\
$\checkmark$ & Privacy \\
\hline \multicolumn{2}{|l}{ Drawbacks } \\
\hline$\times$ & Uncertainty in the final result \\
$\times$ & Limited ancillary services provision \\
$\times$ & Necessity of forecasting the reaction of consumers \\
$\times$ & Avalanche effects or simultaneous reactions may happen \\
\hline
\end{tabular}

One of the most important advantages of decentralized control over centralized one is the scalability. That is, as the number of PEVs increases, it can be difficult to a central controller the management of all PEVs under its governance and the required data for their operation. This aspect is especially important in control methods based on optimized algorithms, because computation time of an optimization algorithm, with a large amount of variables, can be very high. Thus, decentralized control can be more suitable for managing the charging process of multiple PEVs.

Following, the most outstanding characteristics of the droop based controls and the optimal decentralized control are presented. Additionally, different algorithms developed by researchers are included and analyzed.

\section{Droop based control}

A local voltage control can be implemented using droop control methods. Usually, PEVs are individually connected in the low voltage networks where $\mathrm{R} / \mathrm{X}$ ratio is higher than in $\mathrm{MV}$ and $\mathrm{HV}$ distribution networks. Therefore, it can be more efficient to apply the active power control by reducing the power demand of PEVs and even injecting power into the grid [6], [7]. In addition, the primary frequency control is also possible by using the mentioned droop control. A primary frequency control based on the droop method consists in adjusting power demand of the PEVs.

In the droop control for PEVs, a dead zone should be added, where PEVs do not respond to changes in frequency to ensure the longevity of batteries. This dead zone and the slope of the droop control should be defined according to the characteristics of the distribution network where the PEVs are connected and taking into account the willingness of users to participate in system frequency regulation. Finally, there must be an offset that represents the rated power consumption of the PEVs 
when the system is operating without frequency deviations. Thus, for frequency deviations greater than the dead zone, PEV's battery will respond as defined in droop control. If frequency decreases, the battery consumption will be reduced in the first instance. If still this action is not enough, the battery will start to inject power into the grid. Conversely, if frequency increases, the battery consumption will increase, in an attempt to drain the power excess in the system. A droop frequency characteristic including the mentioned improvements is shown in Figure 2.

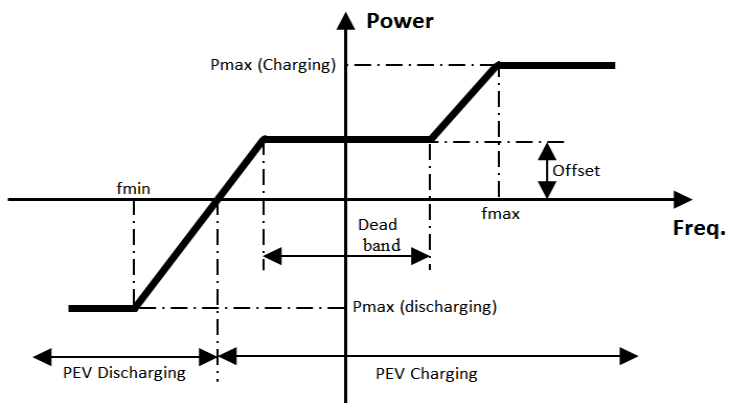

Figure 2. Frequency droop control with V2G [8]-[10]

Many authors have used this classical technique of control. In [11], the droop control method is used to achieve suppression of frequency and voltage fluctuation, without using any type of communication. Possibilities of droop control to maximizes intermittent RES integration, using PEVs and $\mathrm{V} 2 \mathrm{G}$ in islanded grids is researched in [8]. Also, in [9] $\mathrm{V} 2 \mathrm{G}$ is used to provide frequency regulation in a islanded power system with high penetration of wind power sources. Katarina et al. [12] have implemented a reactive power control (RPC) based on droop control method. Results obtained by the researches show that RPC method improves voltages profile while energy losses do not vary significantly. Frederik Geth et al. [13] have proposed an unidirectional (no V2G) droop method in order to limit under-voltage problems that can arise in LV distribution networks.

A similar grid than in [13] is also used in [14]. In this case, authors add a coordinated charging to the droop control. This coordinated charging is based on charging at the minimum charging power to meet the user requirements regarding to departure time. Four cases were analyzed: uncoordinated charging, uncoordinated charging with droop control, coordinated charging and coordinated charging with droop control.

Other possibility is the adaptive droop method, which can offer interesting solutions as in [10], where primary frequency control (PFC) is performed while required charging level of customers is fulfilled. Droop based PFC has the drawback that charging power depends on the frequency level. The authors of this paper address this problem proposing a decentralized V2G control (DVC) which consists in two different smart charging approaches. The first one, called battery SOC holder (BSH), consists in maintaining a minimum SOC during the PFC, pre-selected by the user. The second one, called charging with frequency regulation (CFR), is used to charge the PEV without stopping the frequency regulation. According to the authors, DVC can provide primary frequency regulation while charging demand of PEV users is satisfied.

A drawback of all these droop methods is that, from the standpoint of the network, the effective gain of this method is affected by the number of connected PEVs. Therefore, it is necessary that the control system updates the gain of the droop control of each PEV, which participate on the primary frequency/voltage regulation, to achieve a constant effective gain and avoid fluctuations. In addition, droop control methods alone do not provide very important advantages that other "optimized" methods give, such as load leveling, peak shaving, energy loss reduction, congestion management and charging cost reduction. Thus, in the following section, the most relevant optimized algorithms proposed are presented.

\section{Optimal control}

Optimal control is a technique used to find the best solution from a set of possible solutions or alternatives. This mathematical method is widely used in economics, manufacturing and engineering. Considering electric engineering, mathematical optimization is used in applications such as: power system planning, economic dispatch, unit commitment, network reconfiguration for losses reduction, etc. [15]. In general, a mathematical optimization seeks to find the maximum or the minimum of an objective function, also known as cost function, taking into account several constraints which delimit the number of possible solutions. In the case of PEVs integration, these constraints are user's preferences or technical requirements to be met by the optimized algorithm.

Furthermore, implementation and organization of a decentralized control architecture, where intelligence is distributed in each PEV, can be performed using a tool known as multi-agent system (MAS) [16]. A multi-agent system is a set of two or more intelligent entities, named agents, which interact in an environment. The purpose of this tool is to reduce the complexity of a problem, by dividing it into sub-problems.

Some optimal proposed solutions use price signals. In this approach, on the one hand, PEVs seek to minimize their charging cost. On the other hand, electricity prices are iteratively updated to achieve certain objectives such as avoid network congestions or improve load factor. Figure 3 shows a day-ahead operation of a MAS, which uses price signals strategy. In this solution four different agents play their own role: PEV agent tries to minimize its charging cost and calculates the vehicle charging profile (VCP), the FO agent aggregates all PEVs charging profiles (ACP), the distribution system operator (DSO) is responsible for the correct operation of the distribution network and the market operator (MO) agent updates the electricity prices to influence in the charging profile of the PEV agents. 


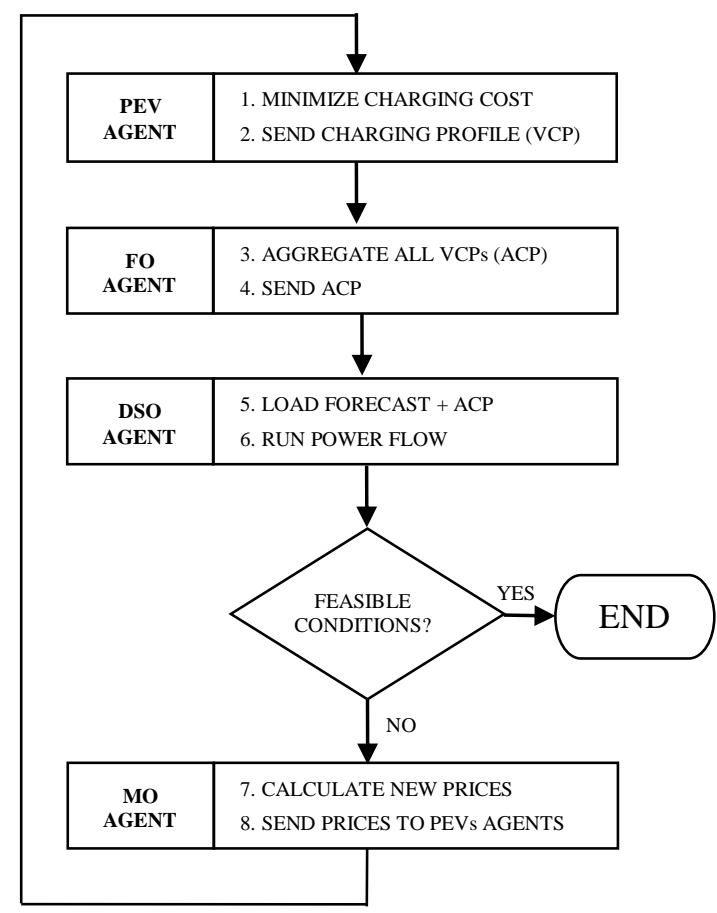

Figure 3. Price signal strategy basic operation using MAS

Several methods are based on this control scheme. Hu et al. have proposed a MAS based on price signals [17]. A DSO agent performs load flows in order to know if there is any congestion problem. If yes, researchers use shadow prices to modify the charging profile of the PEVs. This way, congestion issues can be avoided. Z. Ma et al. [18] have developed an algorithm based on a finitehorizon non-cooperative dynamic game. In this case, each PEV agent tries to minimize its charging cost and the cost incurred in deviating from the average behavior of the PEVs population. After the optimization is performed, the charging profile is sent to the utility which will update the price signal. This process continues until convergence is achieved, with the help of the mentioned penalty term.

In a similar way, Lingwen Gan et al. [19] have proposed an iterative algorithm that tries to fill the off-peak hours optimally. The valley-filling effect is achieved by an iterative optimization process. First, the utility will broadcast a control signal. This control signal is the sum of the forecasted base load and the PEVs power demand. Similarly, in [20] an algorithm is formulated to minimize the overall charging cost of all PEVs, using a game theoretic approach and focusing in distribution networks. In this case, a complex cost function is developed including the generation capacity.

Karfopoulos et al. [21] have designed and modelled a MAS method for charging PEVs. The scheme is similar than the previous analyzed papers. In this solution, a regional aggregation unit agent updates the pricing policy in order to reflect the load level of the distribution transformer. This way, overloading can be limited. A real-time multi-agent system based on price signals is carried out and demonstrated experimentally at laboratory scale in [22]. Four different agents are defined: DSO agent, coordinator agent, local area agent and PEV agent. The PEV agent sends the PEV owner preferences and receives charging set-points from local area agent that had been previously calculated by minimizing a cost function. Coordinator agent aggregates the demand of the local area agents and sends it to the DSO agent, which is responsible for the safely operation of the distribution network. The DSO will perform a power flow in order to know whether there is any congestion. If yes, a virtual price is added to the hours when congestions happen.

An approach based on congestion pricing, in the Internet traffic control, is used in [23] to develop a distributed demand response algorithm, with PEVs in a residential scenario. Price of energy in a certain period of time depends on the aggregated demand. Moreover, each user agent declares a price per time slot that he is willing to pay (WTP), which is an indicator of the requested quality of service. As a consequence of different WTPs between users, load leveling and peak saving can be achieved.

Besides price signals, there are other interesting approaches. In [24] authors have presented a random access framework to schedule the PEVs charging in order to avoid network congestions, as well as voltage drops problems. A network control center monitors the load and voltage parameters of the different buses. When a $\mathrm{PEV}$ is plugged into a specific bus, a smart agent which makes decisions for the PEV will request data from the control center. The smart agent will schedule the PEV charging in function of two stochastic probabilities: access and suspend probability. The first one is designed to prevent the access to the network or resume the charging process, and the second one to suspend charging if any problem exists in the network.

In [25] a stochastic process is also used. Authors have designed a Markov chain to model user driving patterns. The probability of the vehicle to be used is considered in the decision process that determines when a PEV has to be charged, in order to minimize charging costs. A penalty term is introduced to model the flexibility level that a PEV user has. Besides, two versions are analyzed: unidirectional and bidirectional (V2G). A stochastic dynamic programming is used to determine the optimal charging profile.

In addition, decentralized voltage control is introduced in [26], using an iterative algorithm called best response dynamics (BRD) based on non-cooperative game theory. Authors use a sensitivity matrix to evaluate the alterations in voltages values of pilot nodes induced by changes in active and reactive power injections. The solution procedure is as follows: all PEVs send their charging profiles to an aggregator which calculates the voltage on all the pilot nodes (according with the charging profiles of the PEVs) and sends these data back to each PEV. After that, each PEV updates its charging profile to minimize an objective function. Two of them are proposed, minimize the voltage deviations of all pilot nodes (global approach) or minimize the voltage 
deviations of only neighborhood pilot nodes (local approach).

Optimal charging control is generally designed to avoid network congestions and/or improve load factor. In this aspect, price signals are considered a very good approach. In order to know if the MV/LV distribution network will present any congestion issue, some researchers use power flow calculations. However, this option may present problems because sometimes it requires forecasting not only the load demand but also the PEVs load demand, which will be more problematic. In addition, price signals based approaches may increase charging cost for PEVs users, especially in highly loaded distribution networks. Besides, almost no reviewed solutions cope with voltage deviations or unbalances. Table 2 shows the main objectives and the most used tools to integrate PEVs in MV/LV distribution networks.

Table 2. Objectives and tools used to integrate PEVs into distribution networks

\begin{tabular}{ll}
\hline Objective & Most used tools \\
\hline Frequency regulation & Droop control \\
\hline Voltage regulation & Droop control \\
\hline Charging cost minimization & Mathematical optimization \\
& MAS \\
\hline Load leveling & Mathematical optimization \\
& Price signals \\
& MAS \\
\hline Avoid overload issues & Mathematical optimization \\
& Price signals \\
& MAS \\
& Stochastic algorithms \\
& Power flows \\
\hline
\end{tabular}

\section{Integration of PEVs in microgrids}

PEVs can play an important role inside microgrids (MG). Thus, several authors have analyzed how to integrate PEVs within a MG. In [27] and [28] an architecture for the management of PEVs in a microgrid is described. In this case, the element called microgrid central controller (MGCC) is responsible for the market participation of the microgrid, through the market operator (MO), to operate the microgrid in an optimal way. The vehicle controller (VC), the micro-source controller (MC) and the load controller (LC) are located at the field level. The control system may be centralized or decentralized. The performance of the proposed algorithm was tested in a 24 node LV distribution network. A small number of PEVs (8 vehicles) were integrated in the network. Six of them were charged through a single-phase electric vehicle supply equipment (EVSE), while in the other two PEVs three-phase EVSEs were used. According to authors, the load curve was flattened from uncontrolled charging.

Authors of [29] have proposed a droop control approach to reduce frequency and voltage deviations in islanded microgrids. In this control method, the measurement of frequency and voltage at PEVs connection points are carried out to define two droop controls. As commented before, in $\mathrm{LV}$ networks, the $\mathrm{R} / \mathrm{X}$ ratio is high and, therefore, controlling reactive power supply of PEVs could not be effective enough to limit voltage deviations. Thus, injecting or absorbing active power is more efficient to reduce those voltage deviations. Therefore, authors use both droop controls to define the active power of the corresponding PEV. The priority between the two droop control methods is given to frequency regulation, which is more important to maintain microgrid stability, especially when it works in islanded mode.

Decentralized MAS is used in [30] to find optimal active power set-points for PEVs, distributed energy resources and loads. In the proposed system, the PEVs agent transmits the EVSE capacity, the connection time, the initial and the final SOC to an external agent (called as optimizing agent) which is in charge of minimizing the objective function based on load variance and generation cost. Authors use a meta-heuristic solver (AIS) to solve this optimization problem. Once calculation is finished, the optimizing agent sends the optimum set-points to each distributed energy resource, load and PEV.

Authors of [31] present a strategy for congestion management in MGs with PEVs. This methodology is also based on decentralized MAS architecture. Each agent solves an optimization problem to obtain the power dispatch needed to maximize its profits. After an auction, a power flow and an optimal power flow are carried out to check whether the final situation is technically feasible, regarding to overloaded lines. If not, the demand of the specified nodes will be reduced or increased iteratively for each overloaded line, through changes in the PEVs set-points, until congestion problem is cleared.

The integration of PEVs into microgrids could improve their reliability. In addition, PEVs can increase the economic efficiency of electric microgrids by performing a demand side management.

\section{Conclusion}

Decentralized control architecture could be more adequate to manage a large number of PEVs. In this approach, users keep the charging control of their PEVs. However, there are methods to influence indirectly the charging process of the PEVs. In this context, droop control will modify the charging set-point according to voltage and/or frequency level measured at connection point. Although the droop control methods will improve the PEVs integration, there are some issues that should be addressed, such as voltage fluctuations due to the lack of coordination between PEVs. In addition, these droop methods cannot accomplish important tasks such as load leveling, peak saving, congestion management, charging cost reduction, etc. These objectives can be achieved by using optimal control.

One of the most used strategies in optimal control methods is price signals. Researches who use signal prices link electricity prices with energy demand or network capacity. Although it is an interesting approach, linking electricity prices with the energy demand volume 
or network capacity will lead to changes in electricity price formation. Additionally, this operation scheme may induce different electricity prices between different regions. In contrast to droop methods, almost no optimal control deals with voltage deviations and unbalances.

Mixing both control methods can be an interesting approach. However, it can be difficult unless they are focused on managing different variable controls, such as using active power for optimized algorithms and reactive power for droop control methods. This approach should be investigated more in deep.

\section{Acknowledgement}

The work presented in this paper has been supported by a grant of the Basque Government (PRE-2013-2-392) and the University of the Basque Country - UPV/EHU (PES12).

\section{References}

[1] E. V. Landscape, "Global EV outlook for 2020," 2013. [Online]. Available: http://www.iea.org/publications/globalevoutlook_2013.pdf. [Accessed: 12-Jan-2015].

[2] K. Clement-Nyns, E. Haesen, and J. Driesen, "The Impact of Charging Plug-In Hybrid Electric Vehicles on a Residential Distribution Grid," IEEE Trans. Power Syst., vol. 25, no. 1, pp. 371-380, Feb. 2010.

[3] Q. Wu, A. H. Nielsen, J. Ostergaard, S. T. Cha, and Y. Ding, "Impact study of electric vehicle (EV) integration on medium voltage (MV) grids," in 2011 2nd IEEE PES International Conference and Exhibition on Innovative Smart Grid Technologies, 2011, no. 081216, pp. 1-7.

[4] R. C. Green, L. Wang, and M. Alam, "The impact of plug-in hybrid electric vehicles on distribution networks: A review and outlook," Renew. Sustain. Energy Rev., vol. 15, no. 1, pp. 544-553, Jan. 2011.

[5] M. D. Galus, M. G. Vayá, T. Krause, and G. Andersson, "The role of electric vehicles in smart grids," Wiley Interdiscip. Rev. Energy Environ., vol. 2, no. 4, pp. 384-400, Jul. 2013.

[6] M. Rivier, T. Gómez, R. Cossent, and I. Momber, "MERGE Project - D5.1 - New actors and business models for the integration of EV in power systems," 2011.

[7] F. Marra, G. Y. Yang, Y. T. Fawzy, C. Traeholt, E. Larsen, R. Garcia-Valle, and M. M. Jensen, "Improvement of Local Voltage in Feeders With Photovoltaic Using Electric Vehicles," IEEE Trans. Power Syst., vol. 28, no. 3, pp. 35153516, Aug. 2013.

[8] J. A. P. Lopes, P. M. Rocha Almeida, and F. J. Soares, "Using vehicle-to-grid to maximize the integration of intermittent renewable energy resources in islanded electric grids," in International Conference on Clean Electrical Power, 2009, pp. 290-295.

[9] J. R. Pillai and B. Bak-Jensen, "Vehicle-to-Grid for islanded power system operation in Bornholm," in IEEE PES General Meeting, 2010, pp. 1-8.

[10] H. Liu, Z. Hu, Y. Song, and J. Lin, "Decentralized Vehicleto-Grid Control for Primary Frequency Regulation Considering Charging Demands," IEEE Trans. Power Syst., vol. 28, no. 3, pp. 3480-3489, Aug. 2013.

[11] M. Tokudome, K. Tanaka, T. Senjyu, A. Yona, T. Funabashi, and C.-H. Kim, "Frequency and voltage control of small power systems by decentralized controllable loads," in International Conference on Power Electronics and Drive Systems, 2009, pp. 666-671.

[12] K. Knezovic, M. Marinelli, R. J. Moller, P. B. Andersen, C. Traholt, and F. Sossan, "Analysis of voltage support by electric vehicles and photovoltaic in a real Danish low voltage network," in 49th International Universities Power Engineering Conference, 2014, pp. 1-6.
[13] F. Geth, N. Leemput, J. Van Roy, J. Buscher, R. Ponnette, and J. Driesen, "Voltage droop charging of electric vehicles in a residential distribution feeder," in $3 r d$ IEEE PES Innovative Smart Grid Technologies Europe, 2012, pp. 1-8.

[14] N. Leemput, F. Geth, J. Van Roy, A. Delnooz, J. Buscher, and J. Driesen, "Impact of Electric Vehicle On-Board SinglePhase Charging Strategies on a Flemish Residential Grid," IEEE Trans. Smart Grid, vol. 5, no. 4, pp. 1815-1822, 2014.

[15] M. O. W. Grond, N. H. Luong, J. Morren, and J. G. Slootweg, "Multi-objective optimization techniques and applications in electric power systems," in 47th International Universities Power Engineering Conference (UPEC), 2012, pp. 1-6.

[16] S. D. J. McArthur, E. M. Davidson, V. M. Catterson, A. L. Dimeas, N. D. Hatziargyriou, F. Ponci, and T. Funabashi, "Multi-Agent Systems for Power Engineering ApplicationsPart I: Concepts, Approaches, and Technical Challenges," IEEE Trans. Power Syst., vol. 22, no. 4, pp. 1743-1752, Nov. 2007.

[17] J. Hu, A. Saleem, S. You, L. Nordström, M. Lind, and J. Østergaard, "A multi-agent system for distribution grid congestion management with electric vehicles," Eng. Appl. Artif. Intell., vol. 38, pp. 45-58, 2015.

[18] Z. Ma, D. Callaway, and I. Hiskens, "Decentralized charging control for large populations of plug-in electric vehicles," in 49th IEEE Conference on Decision and Control, 2010, pp. 206-212.

[19] L. Gan, U. Topcu, and S. H. Low, "Optimal Decentralized Protocol for Electric Vehicle Charging," IEEE Trans. Power Syst., vol. 28, no. 2, pp. 1-12, 2012.

[20] A. Sheikhi, S. Bahrami, A. M. Ranjbar, and H. Oraee, "Strategic charging method for plugged in hybrid electric vehicles in smart grids; a game theoretic approach," Int. J. Electr. Power Energy Syst., vol. 53, pp. 499-506, Dec. 2013.

[21] E. L. Karfopoulos and N. D. Hatziargyriou, "A Multi-Agent System for Controlled Charging of a Large Population of Electric Vehicles," IEEE Trans. Power Syst., vol. 28, no. 2, pp. 1-1, 2012.

[22] I. Grau Unda, P. Papadopoulos, S. Skarvelis-Kazakos, L. M. Cipcigan, N. Jenkins, and E. Zabala, "Management of electric vehicle battery charging in distribution networks with multiagent systems," Electr. Power Syst. Res., vol. 110, pp. 172179, May 2014.

[23] Z. Fan, "A Distributed Demand Response Algorithm and Its Application to PHEV Charging in Smart Grids," IEEE Trans. Smart Grid, vol. 3, no. 3, pp. 1280-1290, Sep. 2012

[24] K. Zhou and L. Cai, "Randomized PHEV Charging Under Distribution Grid Constraints," IEEE Trans. Smart Grid, vol. 5, no. 2, pp. 879-887, Mar. 2014.

[25] E. B. Iversen, J. M. Morales, and H. Madsen, "Optimal charging of an electric vehicle using a Markov decision process," Appl. Energy, vol. 123, pp. 1-12, Jun. 2014.

[26] O. Beaude, Y. He, and M. Hennebel, "Introducing decentralized EV charging coordination for the voltage regulation," in IEEE PES ISGT Europe, 2013, pp. 1-5.

[27] E. L. Karfopoulos, P. Papadopoulos, S. Skarvelis-Kazakos, I. Grau, L. M. Cipcigan, N. Hatziargyriou, and N. Jenkins, "Introducing electric vehicles in the microgrids concept," in 16 th International Conference on Intelligent System Applications to Power Systems, 2011, pp. 1-6.

[28] J. A. P. Lopes, F. J. Soares, and P. M. R. Almeida, "Integration of Electric Vehicles in the Electric Power System," Proc. IEEE, vol. 99, no. 1, pp. 168-183, Jan. 2011.

[29] J. A. P. Lopes, S. A. Polenz, C. L. Moreira, and R. Cherkaoui, "Identification of control and management strategies for LV unbalanced microgrids with plugged-in electric vehicles," Electr. Power Syst. Res., vol. 80, no. 8, pp. 898-906, Aug. 2010.

[30] B. Ramachandran, S. K. Srivastava, and D. A. Cartes, "Intelligent power management in micro grids with $\mathrm{EV}$ penetration," Expert Syst. Appl., vol. 40, no. 16, pp. 66316640, Nov. 2013

[31] M. A. López, S. Martín, J. A. Aguado, and S. de la Torre, "V2G strategies for congestion management in microgrids with high penetration of electric vehicles," Electr. Power Syst. Res., vol. 104, pp. 28-34, Nov. 2013. 